\title{
Novel Face-Name Paired Associate Learning and Famous Face Recognition in Mild Cognitive Impairment: A Neuropsychological and Brain Volumetric Study
}

\author{
Satyan Nanda ${ }^{a}$ Nandini Mohanan ${ }^{a}$ Sheela Kumaria, b \\ Mridula Mathew $^{a}$ Sushama Ramachandran ${ }^{a}$ P.G. Rajesh Pillai ${ }^{a}$ \\ Chandrasekharan Kesavadas $^{b}$ Sankara Sarma P. ${ }^{c}$ Ramshekhar N. Menon ${ }^{a}$ \\ ${ }^{a}$ Cognition and Behavioral Neurology Subsection, Department of Neurology, Sree Chitra \\ Tirunal Institute for Medical Sciences and Technology, Trivandrum, India; ${ }^{b}$ Department of \\ Imaging Sciences and Interventional Radiology, Sree Chitra Tirunal Institute for Medical \\ Sciences and Technology, Trivandrum, India; ${ }^{C}$ Achutha Menon Centre for Health Science \\ Studies, Sree Chitra Tirunal Institute for Medical Sciences and Technology, Trivandrum, India
}

\section{Keywords}

Mild cognitive impairment - Alzheimer's disease - Volumetric analysis - Face-name pair associate learning · Famous personalities test · Neuropsychology

\section{Abstract}

Purpose: To assess visual associative learning and famous face recognition ability among subjects with stable amnestic mild cognitive impairment $(\mathrm{MCl})$ relative to early stage dementia due to Alzheimer's disease (AD) and cognitively normal healthy controls (NC) and to correlate these differences with volumetric changes on MRI. Methods: A hospital-based cross-sectional observational study was conducted on 61 participants. The subjects underwent neuropsychological evaluation, including validated newly designed tests for novel face-name paired association learning recall and famous face recognition. MRI volumetry was done on a subset of patients to ascertain the topographical patterns of volume loss. Results: There were significant differences in performance on free recall for face-name paired associate learning in $\mathrm{MCl}(n=22)$ compared to NC $(n=20)(p<0.001)$ and $\mathrm{MCl}$ compared to $\mathrm{AD}(n=19 ; p<0.001)$. Significant differences were also noted in scores on the famous personalities test between $\mathrm{MCl}$ and $\mathrm{NC}(p=0.007)$, and $\mathrm{MCl}$ and $\mathrm{AD}(p=0.032)$. The free recall component of face-name pair associative learning significantly correlated with left cuneus ( $p=0.005 ; r=0.833)$ and right cuneus ( $p=0.003 ; r=0.861$ ) volume in AD with no significant correlation among $\mathrm{MCl}$ and NC cohorts. Conclusions: Novel and semantically familiar face-name associative recalls are significantly impaired in $\mathrm{MCl}$, and these potentially predate the $\mathrm{MRI}$ volumetric changes in $\mathrm{MCl}$. Our findings expand the spectrum of recall deficits in $\mathrm{MCl}$. (c) 2019 The Author(s) 


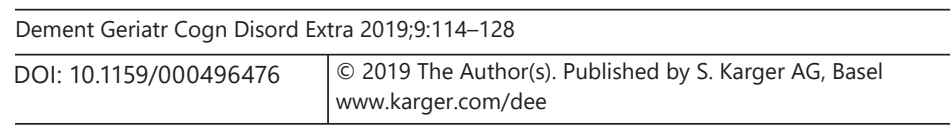

Nanda et al.: Novel Face-Name Paired Associate Learning and Famous Face Recognition in $\mathrm{MCl}$

\section{Introduction}

The prevalence of MCI (mild cognitive impairment) in subjects aged 70-89 years is estimated to be approximately $16 \%$ [1]. From a neuropsychological perspective, the goal is to identify cognitive domains and specific measures that can identify cognitively intact individuals at increased risk for subsequently developing MCI due to Alzheimer's disease (AD). Episodic memory tasks (particularly delayed recall) have generally been considered the most consistent cognitive measure predictive of progression from age-appropriate memory performance to MCI and AD [2-4]. However, it has become increasingly evident that the preclinical period is also characterized by prominent difficulties in other cognitive domains including executive functions (attention), language, and working memory [5]. Studies have indicated that combining episodic memory tasks with language and executive function assessment is of value in identifying MCI at risk for progression to AD dementia [6]. Identifying MCI patients allows for monitoring progression, provides opportunity for appropriate counselling and offers a possible therapeutic window for intervention in the future.

In general, people have good memory for faces compared to other visual stimuli, and the eyes are particularly useful in helping us to remember faces. Subjective reporting of difficulty in remembering names of familiar people is common among subjects with $\mathrm{MCI}$ without overt difficulty in recognition of famous people. These aspects have however not been systematically studied in the MCI literature. Existing tests available have not been tailored to the Indian population and are available with mostly Western faces and names. A recent study indicated the deficits in the ability to learn novel face-name pairs among subjects with MCI and subjective cognitive impairment [7]. Forming an association between a random name and a face is a particularly difficult associative memory task, because names and faces are inherently semantically unrelated across verbal-visual domains, respectively, and have to be learnt. In addition to the hippocampus, the encoding of novel face-name associations likely requires the integration of several other brain regions, including the prefrontal and fusiform cortices [8]. In studies involving novel face-name pairs, it has been found that this encoding is subserved by a distributed functional network of brain regions, including the hippocampal formation, dorsolateral prefrontal cortex, pulvinar nucleus of the thalamus, fusiform gyrus, and adjacent areas of the visual association cortex [9].

It has been shown that MCI subjects at risk for subsequent development of dementia were impaired on tests of naming for faces and buildings as compared to naming non-living objects relative to healthy controls. Proper names are thought to be more difficult to retrieve than common nouns due to the weak and arbitrary links between a proper noun and its reference [10].

We hypothesize in this study that visual paired associate learning and famous face recognition ability are impaired in patients with amnestic MCI in comparison to cognitively normal healthy controls (NC), as in patients with established early AD. We also attempt to correlate these differences with atrophy in specific ROIs as ascertained by volumetric studies. The lack of any similar studies with utilization of Indian rather than Western faces is another novel attempt in our study.

\section{Materials and Methods}

\section{Study Design and Setting}

The study was designed as a hospital-based cross-sectional observational study. The cases were selected from among patients with cognitive deficits attending the outpatient general neurology and neurobehavioral-disorders clinic of our tertiary referral hospital. 
Nanda et al.: Novel Face-Name Paired Associate Learning and Famous Face

Recognition in $\mathrm{MCl}$

Inclusion Criteria

The lower limit for age was taken as 55 years and the upper limit was selected as 85 years. A minimum of 6 years of formal education was a prerequisite. Subjects were required to be on stable doses of medications for cognition or behaviour for at least 2 months prior to screening as it is well known that medications such as cholinesterase inhibitors may influence the final neuropsychology test performances. The Hospital Anxiety and Depression Scale score was required to be $<7$. Cases were classified as amnestic MCI based on the Petersen criteria [11] with Addenbrooke's Cognitive Examination-Malayalam (M-ACE) score ranging between 75 and 90 and a Clinical Dementia Rating (CDR) score of $\leq 0.5$ [12]. Early AD diagnosis was based on and should have met the National Institute on Aging and Alzheimer's Association criteria for probable AD [13], M-ACE [14] score ranging from 70 to 85, and a CDR score of $0.5-1$. NC were enrolled if they had no self-declared impairment on cognitive or behavioural functions and if they had M-ACE score $>85$.

Exclusion Criteria

CDR $>1$; any other major psychiatric or neurological disorders known to affect cognitive function or which would make it difficult for the subject to comply with study requirements in the long term; uncontrolled hypertension, diabetes, coronary artery, cerebrovascular disease, major organ diseases, head injury, drug abuse, psychiatry medication use including antidepressants, history suggestive of obstructive sleep apnoea; subjects who could not follow the study protocol for physical (vision; hearing) reasons; subjects with contraindications to MRI (e.g., pacemakers, artificial heart valves, etc.); subjects who had evidence of extensive white matter changes on MRI (Fazeka's grade II and above).

\section{Methodology}

Those subjects consenting for the study were interviewed with a structured proforma for details of age and mode of onset of cognitive decline along with a relevant history. Consecutive patients presenting to the respective clinics during the study period were selected of whom around $40-50 \%$ were expected to be women. No sampling method was used. All the cases were subject to clinical and neuropsychological evaluation. MRI of the brain with volumetry analysis was done for the subjects who gave their consent for the same.

Standard vernacular adaptations of neuropsychological tools for the diagnosis of MCI were as listed below:

1 M-ACE (Addenbrooke's Cognitive Examination) validated for use in the Malayalamspeaking population $[14,15]$

2 Rey Auditory Verbal Learning test (RAVLT learning and delayed recall scores) $[15,16]$

3 Wechsler memory scale (WMS): visual and verbal subsets [15, 17]

4 Wisconsin card sorting test $[15,18]$

5 Semantic battery [19]

6 Trail making test A and B $[15,20]$

7 Warrington face recognition test [21]

8 Visual Object and Space Perception Test [22]

\section{Test Development for Face-Name Pair Association}

The test for face-name pair association had to be devised as no such test validated for use in a South Indian population cohort exists. A similar test was devised for the English-speaking population and was used in an American cohort for assessing the connectivity changes in MCI subjects after giving them explicit memory training using face-name pairing [23]. The methodology for tool development for validation in our cohort was as implemented in the FNAME study [24], and the flow diagram to depict the order of test implementation is depicted in Figure 1. 
Fig. 1. Workflow in the cohort depicting the protocol of implementation of neuropsychology tests. VOSP, Visual Object and Space Perception Test.

Study cohort (AD, MCl, and controls): $n=61$

Initial assessment:

- ACE-Malayalam

- HADS scale

- CDR

Standardized test:

- RAVLT

- Warrington recognition test for faces

- Trail A and B

- Wisconsin card sorting test

- WMS-digit span

- VOSP

Test for associative learning and famous faces recognition

Devised test:

- Face-name paired associative learning and recall

- Famous personalities recognition test

Test Development

For the evaluation of visual paired associate learning, the following procedure was employed for devising a test:

- 16 random faces were selected from the Internet ( 6 females, 10 males). These faces ranged in age from 20 to 70 years.

- The faces were converted to a greyscale to obviate the effect of colour as cues for face recognition.

- Intimate features such as eyes, facial, cosmetic and dressing/grooming characteristics were retained so as to enable their help (visual clues) in forming an association for the stimulus.

- A gender- and age-appropriate name was assigned to each of the faces. The names chosen were random and covered names from all three prominent communities inherent to the Malayalam-speaking population.

- The name assigned was displayed in English and Malayalam alongside the face in a font size of 44 on standard power point presentation (Appendix 1).

- 16 faces and their corresponding names in 1 set were displayed sequentially for $10 \mathrm{~s}$ each.

- Total time of administration for 1 set: $16 \times 10 \mathrm{~s}=160 \mathrm{~s}$.

- In total, 3 such rounds were administered, in a successive fashion with no intervening waiting time.

- At no time was a verbal stimulus given. The patients had to visually associate the face and names.

- After 20 min following the 3rd round, a free recall (FR) session was conducted with serial isolated display of the initially viewed faces. The maximum time allotted for FR was $10 \mathrm{~s}$ per stimulus. The FR scores were given as 0 or 2 for each stimulus. The cumulative FR scores were calculated for 16 face stimuli and tabulated on an Excel sheet (total score 32).

- After the FR phase was over, cued recall (CR), in which names would be displayed, was conducted. 
Nanda et al.: Novel Face-Name Paired Associate Learning and Famous Face Recognition in $\mathrm{MCl}$

Table 1. Comparison of data between 2 neuropsychologists (means \pm SD)

\begin{tabular}{lll}
\hline Neuropsychology parameters & Neuropsychologist 1 & Neuropsychologist 2 \\
\hline Free recall & $27.20 \pm 6.87$ & $25.45 \pm 6.27$ \\
Cued recall & $15.70 \pm 0.66$ & $15.91 \pm 0.30$ \\
Famous faces score & $27.40 \pm 2.78$ & $27.18 \pm 3.46$ \\
\hline
\end{tabular}

Table 2. Demographic profile of subjects

\begin{tabular}{|c|c|c|c|c|c|c|}
\hline & MCI & $\mathrm{AD}$ & NC & $\begin{array}{l}\text { MCI-NC } \\
\text { ( } p \text { value) }\end{array}$ & $\begin{array}{l}\text { MCI-AD } \\
(p \text { value })\end{array}$ & $\begin{array}{l}\mathrm{AD}-\mathrm{NC} \\
(p \text { value })\end{array}$ \\
\hline Total & 22 & 19 & 20 & - & - & - \\
\hline Males & 15 & 10 & 8 & 0.067 & 0.309 & 0.429 \\
\hline Females & 7 & 9 & 12 & 0.067 & 0.309 & 0.429 \\
\hline Mean age (SD), years & $69.91(7.65)$ & $68.05(6.85)$ & $65.05(6.01)$ & 0.028 & 0.421 & 0.154 \\
\hline Mean years of formal schooling (SD) & $13.36(3.47)$ & $13.19(3.39)$ & $14.36(5.02)$ & 0.487 & 0.877 & 0.456 \\
\hline
\end{tabular}

SD, standard deviation; MCI, mild cognitive impairment; AD, Alzheimer's disease; NC, normal controls. Values in boldface indicate statistically significant $p$ values.

Table 3. Baseline screening test results (means $\pm \mathrm{SD}$ ) for AD, MCI, and NC with $p$ values for group differences

\begin{tabular}{lllrrrr}
\hline $\begin{array}{l}\text { Neuropsychology } \\
\text { parameter }\end{array}$ & AD & MCI & NC & MCI-NC & \multicolumn{1}{c}{$\begin{array}{l}\text { AD-MCI } \\
(p \text { value })\end{array}$} & $\begin{array}{l}\text { AD-NC } \\
(p \text { value })\end{array}$ \\
\hline MMSE & $24.53 \pm 3.02$ & $27.96 \pm 1.59$ & $29.4 \pm 0.91$ & $\mathbf{0 . 0 0 1}$ & $<\mathbf{0 . 0 0 1}$ & $<\mathbf{0 . 0 0 1}$ \\
ACE-total & $74.18 \pm 13.74$ & $83.59 \pm 9.88$ & $93.47 \pm 4.72$ & $\mathbf{0 . 0 0 1}$ & $\mathbf{0 . 0 2 3}$ & $<\mathbf{0 . 0 0 1}$ \\
CDR & $0.62 \pm 0.28$ & $0.48 \pm 0.24$ & $0.13 \pm 0.23$ & $<\mathbf{0 . 0 0 1}$ & 0.103 & $<\mathbf{0 . 0 0 1}$ \\
\hline
\end{tabular}

SD, standard deviation; MCI, mild cognitive impairment; AD, Alzheimer's disease; NC, normal controls; MMSE, Mini Mental State Examination; ACE-total, Addenbrooke's Cognitive Examination-total score; CDR, Clinical Dementia Rating. Values in boldface indicate statistically significant $p$ values.

- The subjects had to choose one among the 3 names which were displayed on the screen along with the target face. These 3 names were phonetically distinct and consisted of one of the names from the stimulus set (correct response) and 2 distracters. The 2 distracters were again randomly selected from common names prevalent among the Malayalam-speaking population.

- The subjects were given 1 point if correct, 0 if incorrect. This gave the CR scores. The cumulative CR scores for each stimulus were tabulated on an Excel sheet (total score 16).

- The time for this exercise was $5 \mathrm{~s}$ per stimulus.

- At the end the sum total was to be done in an Excel sheet.

\section{Procedure of Developing the Famous Personalities Test}

15 random famous personalities were selected from the Internet and constituted famous personalities from different walks of life including politicians, sportspersons, musicians, film actors and social activists. These famous personalities were chosen from a wide range of generations, and most of them had attained prominence before the year 2000. No interna- 
Nanda et al.: Novel Face-Name Paired Associate Learning and Famous Face Recognition in $\mathrm{MCl}$

Table 4. Scores (means \pm SD) in AD, MCI, and NC on associative learning with age-, gender-, and education-adjusted comparisons between the same cohorts

\begin{tabular}{|c|c|c|c|c|c|c|}
\hline $\begin{array}{l}\text { Neuropsychology } \\
\text { parameters }\end{array}$ & $\mathrm{AD}$ & MCI & $\mathrm{NC}$ & $\begin{array}{l}\text { MCI-NC } \\
(p \text { value })\end{array}$ & $\begin{array}{l}\text { AD-MCI } \\
(p \text { value })\end{array}$ & $\begin{array}{l}\mathrm{AD}-\mathrm{NC} \\
\text { ( } p \text { value })\end{array}$ \\
\hline Free recall & $4.58 \pm 4.98$ & $10.86 \pm 5.82$ & $20.20 \pm 5.98$ & $<0.001$ & 0.001 & $<0.001$ \\
\hline Cued recall & $8.74 \pm 3.91$ & $12.86 \pm 2.89$ & $14.35 \pm 1.73$ & 0.061 & $<0.001$ & $<0.001$ \\
\hline
\end{tabular}

SD, standard deviation; MCI, mild cognitive impairment; AD, Alzheimer's disease; NC, normal controls. Values in boldface indicate statistically significant $p$ values.

Table 5. Scores (means \pm SD) in AD, MCI, and NC on famous personalities with age-, gender-, and education-adjusted comparisons between the same cohorts

\begin{tabular}{|c|c|c|c|c|c|c|}
\hline $\begin{array}{l}\text { Neuropsychology } \\
\text { parameter }\end{array}$ & $\mathrm{AD}$ & MCI & $\mathrm{NC}$ & $\begin{array}{l}\text { MCI-NC } \\
(p \text { value })\end{array}$ & $\begin{array}{l}\text { AD-MCI } \\
\text { ( } p \text { value })\end{array}$ & $\begin{array}{l}\mathrm{AD}-\mathrm{NC} \\
(p \text { value })\end{array}$ \\
\hline FP & $19.53 \pm 4.54$ & $22.71 \pm 4.03$ & $26.35 \pm 2.08$ & 0.007 & 0.032 & $<0.001$ \\
\hline
\end{tabular}

AD, Alzheimer's disease; MCI, mild cognitive impairment; NC, normal controls; FP, famous personalities. Values in boldface indicate statistically significant $p$ values.

tional figures of prominence were used in our test. After choosing the famous personalities, a presentation slide was made in which besides the famous personality 2 distracter faces of the same age group and gender were used. The subjects had to correctly identify the famous personality and also had to name them. If they were able to correctly identify the famous personality but unable to name them, they were scored as 1 , and if they were able to name them also, they were scored 2 . If they were unable to identify the famous personality, a score of 0 was given. The time of exposure of the stimulus was fixed at $15 \mathrm{~s}$ per stimulus. If the subjects were not able to answer by the allotted time, a score of 0 was given, and the next stimulus was given without coming back to the previous slide. The procedure mentioned above was done for all the stimuli. At the end a total score was calculated.

\section{Methodology of MRI Acquisition}

This is detailed in Appendix 2.

\section{Statistical Analysis}

Demographic data, neuropsychological measures, and cortical grey matter (GM) volumes between study groups were analysed using the Statistical Package for the Social Sciences Statistics for Windows version 21 (SPSS, IBM, Armonk, NY, USA). As the data contained quantitative continuous variables, the measure of assessment was the mean with standard deviation. The demographic and cognitive variables were compared across the groups: $\mathrm{NC}, \mathrm{MCI}$, and $\mathrm{AD}$, using univariate analysis of variance (ANOVA) after age correction. Pearson's correlation was applied for determining the correlation between volumes and neuropsychology variables. Linear regression analysis was performed to adjust for the effect of age, gender, total intracranial volume, and education in the comparisons between volumes and neuropsychology. In all comparisons, the level of statistical significance was set at $p<0.05$. 
Nanda et al.: Novel Face-Name Paired Associate Learning and Famous Face Recognition in $\mathrm{MCl}$

Table 6. Correlation ( $p$ values; $r$ correlation coefficients) of free recall scores on face-name pair test with ACE-recall, RAVLTdelayed recall, WMS-delayed logical memory, and WMS-delayed visual memory scores in controls, MCI, and AD cohorts

\begin{tabular}{lllll}
\hline & ACE-recall & $\begin{array}{l}\text { RAVLT-delayed } \\
\text { recall }\end{array}$ & $\begin{array}{l}\text { WMS-delayed logical } \\
\text { memory recall }\end{array}$ & $\begin{array}{l}\text { WMS-delayed visual } \\
\text { memory recall }\end{array}$ \\
\hline NC & $\mathbf{0 . 0 3 5 ; 0 . 5 4 6}$ & $\mathbf{0 . 0 0 2 ; 0 . 7 3 2}$ & $\mathbf{0 . 0 2 7} ; \mathbf{0 . 5 8 9}$ & $0.200 ; 0.364$ \\
MCI & $0.199 ; 0.292$ & $\mathbf{0 . 0 1 2 ; 0 . 5 3 6}$ & $0.050 ; 0.433$ & $0.473 ; 0.166$ \\
AD & $\mathbf{0 . 0 1 5 ; 0 . 5 7 7}$ & $\mathbf{0 . 0 3 3 ; 0 . 5 1 8}$ & $\mathbf{0 . 0 0 3 ; 0 . 6 6 8}$ & $0.079 ; 0.438$ \\
\hline
\end{tabular}

MCI, mild cognitive impairment; AD, Alzheimer's disease; NC, normal controls; ACE, Addenbrooke's Cognitive Examination; RAVLT, Rey Auditory Verbal Learning test; WMS, Wechsler memory scale. Values in boldface indicate statistically significant $p$ values and correlation coefficients.

Table 7. Correlation ( $p$ values; $r$ correlation coefficients) of famous personalities scores with Warrington's Faces, RAVLT-delayed recall, and WMS-delayed visual memory scores in NC, MCI, and AD cohorts

\begin{tabular}{llll}
\hline & Warrington's Faces & RAVLT-delayed recall & $\begin{array}{l}\text { WMS-delayed visual } \\
\text { memory recall }\end{array}$ \\
\hline $\mathrm{NC}$ & $0.967 ; 0.052$ & $0.182 ; 0.364$ & $0.719 ;-0.106$ \\
$\mathrm{MCI}$ & $0.076 ; 0.406$ & $\mathbf{0 . 0 2 8 ; 0 . 4 7 9}$ & $\mathbf{0 . 0 3 5 ; 0 . 4 6 2}$ \\
$\mathrm{AD}$ & $0.039 ; 0.505$ & $0.443 ; 0.199$ & $0.293 ; 0.271$ \\
\hline
\end{tabular}

MCI, mild cognitive impairment; AD, Alzheimer's disease; NC, normal controls; RAVLT, Rey Auditory Verbal Learning test; WMS, Wechsler memory scale. Values in boldface indicate statistically significant $p$ values and correlation coefficients.

\section{Ethical Considerations}

This study had the approval of the Institutional Ethics Committee (reference No. IEC/978/2016), and informed consent was obtained from all cases and controls.

\section{Results}

\section{Pilot Study}

The pilot validation study for the 2 newly designed tests was done by 2 neuropsychologists on a total of 21 cognitively normal healthy subjects across different education and age groups who consented for this study. The data parameters are summarized in Table 1 . The unpaired $t$ test did not show any significant difference between the FR ( $p=0.54)$ and CR ( $p=$ 0.36 ) scores among the 2 neuropsychologists.

\section{Demographic Profile}

There were $22 \mathrm{MCI}$ subjects and $19 \mathrm{AD}$ subjects who were enrolled consecutively (Table 2). For comparison 20 healthy controls who were age and gender matched were also included in the analysis. ANOVA for age when comparing MCI versus controls was significant with a $p$ value of 0.028 whereas education was not noted to be significantly different. No other significant differences were evident between the 3 groups. 
Nanda et al:: Novel Face-Name Paired Associate Learning and Famous Face

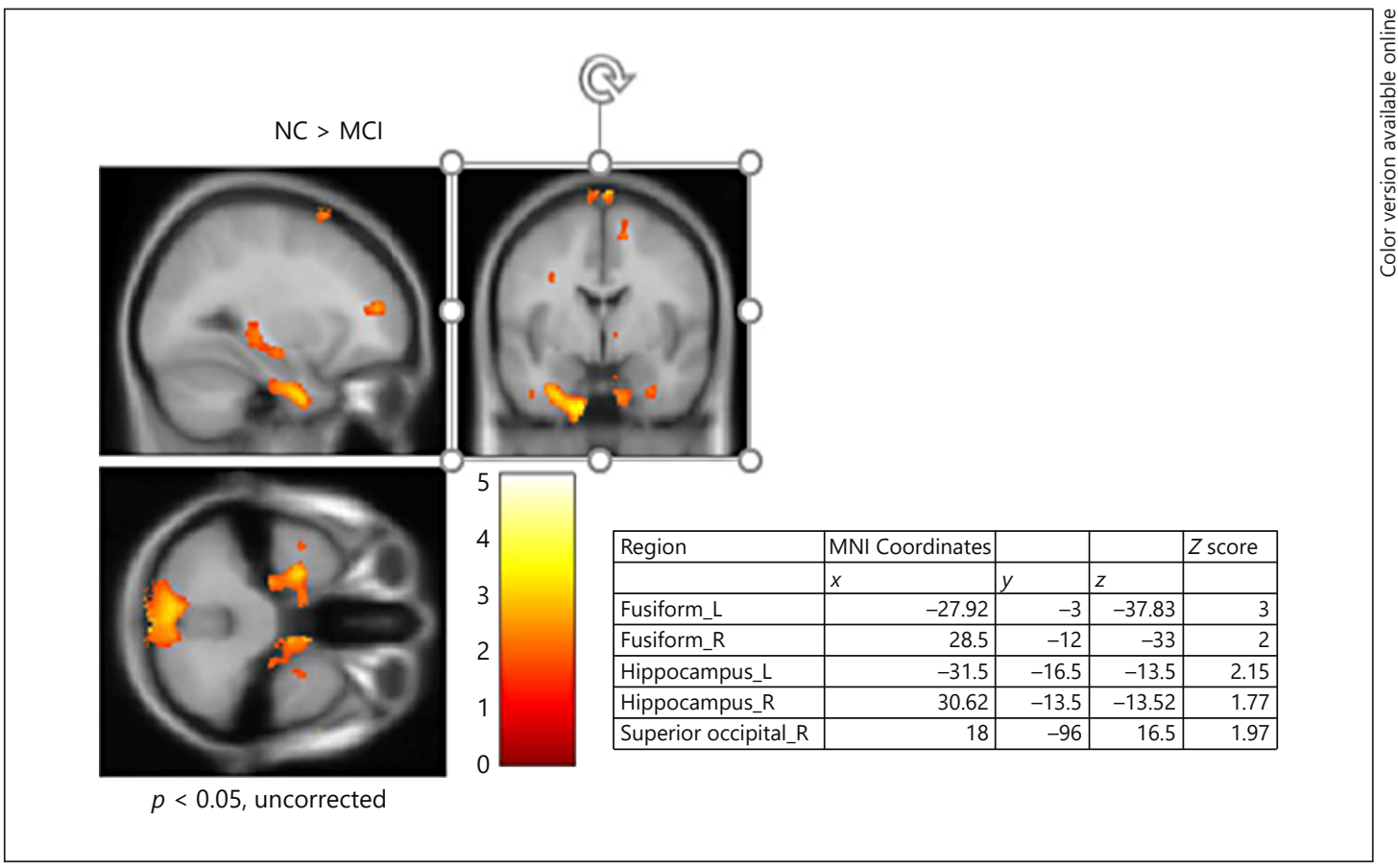

Fig. 2. Voxel-based morphometry analysis between MCI and controls (NC) with age, sex, and total intracranial volume as covariates (without applying family-wise error correction).

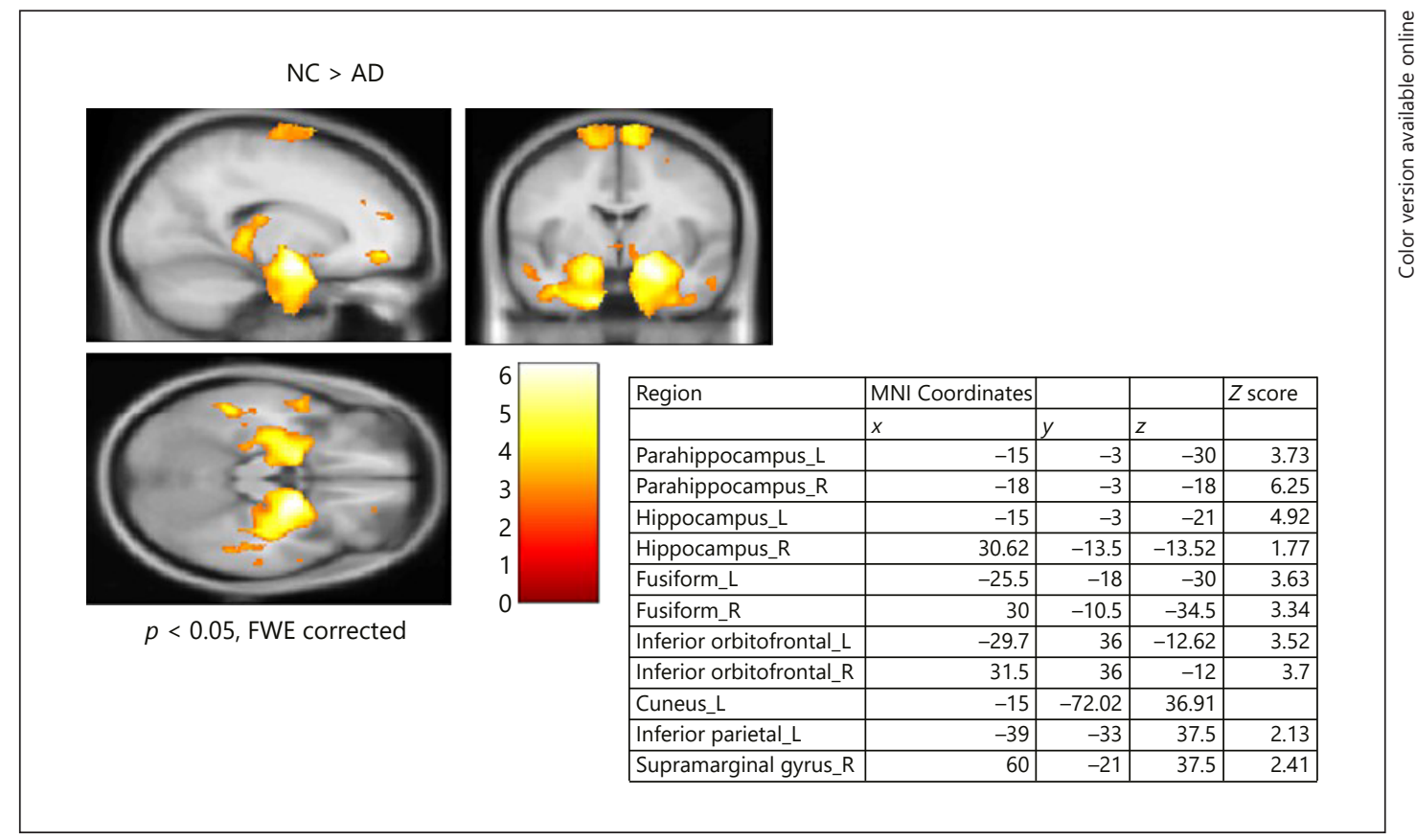

Fig. 3. Voxel-based morphometry analysis between $\mathrm{AD}$ and $\mathrm{MCI}$ with age, sex, and total intracranial volume as covariates. NC, normal controls; FWE, family-wise error rate. 
Nanda et al.: Novel Face-Name Paired Associate Learning and Famous Face

Recognition in $\mathrm{MCl}$

Table 8. Values of volumetric analysis for MCI, AD, and NC as per region of interest (ROI) and age-, gender-, and TIV-adjusted differences between the cohorts as depicted by $p$ values

\begin{tabular}{|c|c|c|c|c|c|c|}
\hline ROI & $\mathrm{AD}(15)$ & MCI (14) & NC (13) & $\begin{array}{l}\text { AD-MCI } \\
(p \text { value })\end{array}$ & $\begin{array}{l}\text { MCI-NC } \\
\text { ( } p \text { value })\end{array}$ & $\begin{array}{l}\text { AD-NC } \\
\text { ( } p \text { value })\end{array}$ \\
\hline TIV & $1,379.8$ (119.51) & $1,347.57(126.13)$ & 1,301.39 (109.69) & 0.499 & 0.321 & 0.084 \\
\hline GM & $411.93(52.80)$ & 369.87 (69.85) & $328.46(53.64)$ & 0.011 & 0.852 & $<0.001$ \\
\hline WM & 538.27 (49.51) & 551.57 (51.21) & $547.23(40.34)$ & 0.013 & 0.836 & 0.001 \\
\hline $\mathrm{CSF}$ & 426.47 (41.57) & $421.71(44.44)$ & $421.85(45.94)$ & 0.189 & 0.763 & 0.027 \\
\hline lHip & $2.74(0.67)$ & $3.44(0.70)$ & $3.68(0.27)$ & 0.006 & 0.346 & $<0.001$ \\
\hline rHip & $2.68(0.74)$ & $3.56(0.70)$ & $3.82(0.25)$ & 0.003 & 0.315 & $<0.001$ \\
\hline lPhg & $3.16(0.47)$ & $3.40(0.50)$ & $3.57(0.33)$ & 0.062 & 0.144 & $<0.001$ \\
\hline rPhg & $3.44(0.48)$ & $3.77(0.44)$ & $3.94(0.39)$ & 0.022 & 0.142 & \\
\hline ICun & $3.19(0.65)$ & $3.05(0.53)$ & $2.98(0.27)$ & 0.445 & 0.801 & 0.770 \\
\hline rCun & $3.44(0.48)$ & $3.32(0.42)$ & $3.46(0.48)$ & 0.960 & 0.106 & 0.244 \\
\hline IPrecun & $5.85(0.79)$ & $6.07(0.78)$ & $5.97(0.81)$ & 0.428 & 0.850 & 0.038 \\
\hline rPrecun & $5.87(1.03)$ & $6.01(0.88)$ & $5.96(0.79)$ & 0.284 & 0.629 & 0.017 \\
\hline lFusG & $6.39(0.99)$ & $6.67(0.92)$ & $6.63(0.58)$ & 0.231 & 0.881 & 0.035 \\
\hline rFusG & $6.41(0.94)$ & $6.90(0.79)$ & $6.85(0.70)$ & 0.019 & 0.966 & 0.002 \\
\hline
\end{tabular}

Means with standard deviations in parentheses. MCI, mild cognitive impairment; AD, Alzheimer's disease; NC, normal controls; TIV, total intracranial volume; GM, grey matter; WM, white matter; CSF, cerebrospinal fluid; lHip, left hippocampal gyrus; rHip, right hippocampal gyrus; lPhg, left parahippocampal gyrus; rPhg, right parahippocampal gyrus; lCun, left cuneus; rCun, right cuneus; IPrecun, left precuneus; rPrecun, right precuneus; lFusG, left fusiform gyrus; rFusG, right fusiform gyrus. Values in boldface indicate statistically significant $p$ values.

\section{Screening Test Results}

The screening test results demonstrated significant differences between the three cohorts in MMSE and ACE total scores (Table 3). Domain-wise differences, especially on memory subsets are depicted in online supplementary Tables 1 and 2 (for all online suppl. material, see www.karger.com/doi/10.1159/000496476).

\section{Test of Face-Name Pair Associative Learning}

Significant impairment was noted on FR scores among MCI and AD subjects relative to NC (Table 4). CR scores failed to demonstrate significant differences between the MCI and NC as opposed to MCI-AD and AD-NC comparisons.

\section{Famous Personalities Test}

Significant impairment was noted among $\mathrm{MCI}$ and $\mathrm{AD}$ subjects on famous personalities test (FP) scores in comparison to NC as well as between AD and MCI (Table 5).

\section{Correlation of Associative Learning Recall with Standard Memory Tests}

The FR scores had a significant positive correlation with RAVLT delayed recall scores for all 3 cohorts (Table 6). Additionally, a significant positive correlation was noted with ACErecall and WMS-delayed logical memory recall scores in NC and AD cohorts but not in the MCI cohort.

Correlations of Famous Personality Scores with Standard Tests

A significant association was noted for FP scores on verbal and visual memory subsets only among MCI subjects (RAVLT-delayed recall $[p=0.028]$ and WMS-delayed visual recall scores $[p=0.035]$ ) (Table 7). 
Nanda et al.: Novel Face-Name Paired Associate Learning and Famous Face Recognition in $\mathrm{MCl}$

\section{Volumetric Analysis (Subset Analysis)}

This analysis was done for 13 controls, $14 \mathrm{MCI}$ subjects and $15 \mathrm{AD}$ subjects as the rest of the subjects did not give a consent for MRI. The comparisons between the different groups are made after adjustment for age, gender, and total intracranial volume of the cohorts.

There was no statistically significant difference found for the tested region of interest (ROI) volumes among the two cohorts of MCI and controls after false detection rate adjustment for the covariates although voxel-based morphometry showed differences on the uncorrected images (Fig. 2). Table 8 depicts the ROIs that demonstrated significant differences between AD-MCI (Fig. 3) and AD-controls following false detection rate correction.

\section{Correlation between Neuropsychology and Volumetry}

All correlations were made after adjusting for age, gender, and education of individual cohort(s). There was a statistically significant correlation observed with white matter ( $p=$ $0.041 ; r=0.686)$, left $(0.005 ; 0.833)$ and right cuneus $(0.003 ; 0.861)$ volumes for FR scores in the AD cohort. No statistically significant correlations were noted between FR, CR, FP recognition scores, and volumes in tested ROIs among the MCI and NC cohorts (additional data in online suppl. Tables 3-5).

\section{Discussion}

Our study indicates pertinent issues with regard to visual associative learning as demonstrated in this study using a novel face-name paired associative learning and delayed visual memory as evident on FP recognition among patients with amnestic MCI. Improvement on cueing of names (verbal cues) points to primarily retrieval deficits in this group of patients and given the profile of early AD patients, failure to improve on cueing may be utilized to categorize amnestic MCI at risk of progression. Additionally, correlations established on MRI volumetry with cuneus volumes in early AD are indicative of visual encoding errors in this group of patients. Absence of correlation in amnestic MCI points towards the utility of facename paired associative deficits as a novel neuropsychological biomarker for amnestic MCI at risk, even prior to the development of structural changes on MRI.

The pilot study for new tests was undertaken to note the reproducibility of results with regard to age, gender, and education differences among the study population. There are no similar tests in the Indian population, and the development of new tests as done by us for visual associative paired learning (face-name pairing) needs to be emphasized. Studies on larger patient-control groups are needed to ascertain the final validity of our tests. The demographic data between MCI, AD, and controls were education matched for all the subcategories being studied. Even after adjustment for age, gender, and education, the performances on neuropsychological tests showed statistically significant differences between MCI and AD cohorts for the tested parameters of FR and CR on the face-name pairing test.

When comparing between MCI and controls on face-name paired associate learning, the FR scores were found to be statistically significant with no significant differences on CR scores. This is in agreement with a recent study which demonstrated significant impairment of tasks involving face-name associative learning in MCI subjects as compared to controls [7]. As compared to our study which utilized previously unpresented novel names as distracters, the study utilized the names presented previously for other faces as distracters. The study did not have a provision for FR, and instead subjects had to choose between the correct name and distracter which were presented (CR). Our test in comparison can be considered to be a comprehensive measurement of associative learning in that there is no associative clue which 
is being given in the 1st attempt thus offsetting the advantage being posed by name (item) familiarity or forced-choice recognition rather than FR. The methodology also differed in our study as we utilized three trials for better chances of recollection of names considering an FR component, and there was a gap of 20 min between the last exposure and FR; the study mentioned above does not have a mention of the same. This was done as we needed to assess delayed recall memory of face-name pair association. In order to test the validity of the new test, the results on FR scores were correlated with another score of delayed recall, namely RAVLT-delayed recall, and as per our results the same was concordant for cohorts of MCI, controls, and AD.

The scores on the FP test were significantly different between MCI and controls, and the $\mathrm{AD}$ and MCI cohort. The test for FP aims to assess recall of famous personalities with autobiographical significance for adults. Thus, this test aimed to test retrograde and visual semantic memory as is applicable in a real-world scenario. Given the fact that most patients with early cognitive impairment report inability to recall names of people who were well known to them in the distant past (despite a preserved ability to recall having met them or their relationship context), we wanted to evaluate this capability specifically in MCI. Our study thus brought about several interesting findings. Our test utilized a bipartite scoring model in which the subject had to first identify the famous personality from among the 2 distracters, and then had to name the person. Thus, this test aimed to identify remote visualverbal recall of names of semantically familiar visual stimuli, i.e. famous faces. These findings are in good agreement with another study wherein it was demonstrated that both MCI and AD subjects performed significantly worse on a graded-faces name test as compared to NC. The test mentioned above utilized famous personalities from the past 5 decades some of whom might have been out of limelight in the recent past [10].

Both the volumetric and group analyses revealed expectedly significant differences between $\mathrm{AD}$ and $\mathrm{MCI}$ with respect to bilateral hippocampus and right parahippocampal gyrus volumes. There was also a significant reduction noted in the volume of the right fusiform gyrus. Furthermore, the reduction in volume of hippocampi was more significant as compared to the reduction in volume of the parahippocampal gyrus. This is in accordance with previous studies which have demonstrated significant reductions in the volume of the hippocampus and parahippocampal cortex in AD patients compared to amnestic MCI subjects. The greater relative reduction of volume of hippocampi (left; $p=0.006$; right; $p=0.003$ ) as compared to reduction in the volume of the parahippocampal gyrus (right; $p=0.022$ ) is also in accordance with the findings by this study [24]. The reduced volume of the fusiform gyrus would be indicative of volume loss spreading to adjacent temporo-occipital association areas with progression of the disease pathology.

In our cohort there were no significant differences found in the volumetry and group analysis of studied regions between amnestic $\mathrm{MCI}$ and $\mathrm{NC}$ in the false detection rate-corrected images on voxel-based morphometry despite differences noted in the uncorrected images. This is in contrast to a study [25] which had demonstrated significant differences in the volumetry of hippocampi and the entorhinal cortex in MCI as compared to NC. The heterogeneity in the degree of cognitive impairment in the MCI cohort being compared here might explain these non-concordant findings. In our MCI cohort, the mean MMSE score was 27.96 as compared to 24 for the study mentioned above. This might imply a relatively advanced stage of MCI or even prodromal AD in the reference cohort. The degree of atrophy in MCI subjects is also expected to progressively increase as the severity of the cognitive impairment increases. Further the limited sample size of our cohorts available for volumetry is a factor which needs to be considered while interpreting our results.

A positive correlation was demonstrated with regard to performance on FR and CR scores with right cuneus volumes in the AD cohort. The FR scores also had a positive significant 
correlation to left cuneus and white matter volumes. In a recent study done to assess the structural brain substrates of face-name associative learning, it was concluded that the same is a higher order cognitive process and requires the activation of multiple brain structures and processes [26]. This finding is corroborated by a functional MRI study in which the facename pairing was shown to activate a widespread cortical network, including medial frontal, left frontal operculum, angular gyrus, left lateral temporal, parietal and occipital cortices [23]. The activation of the striate and extrastriate cortex while encoding for novel face-name pairs has also been demonstrated $[9,27]$. In this context, our findings of correlation of only right and left cuneus volume to visual associative learning tests represent a novel finding which needs to be confirmed by other similar studies and are indicative of visual-encoding/retrieval deficits. With respect to the MCI cohort, no significant correlation could be derived between the associative learning scores and volumetric analysis. It can be concluded in this context that dysfunction on neuropsychology testing may predate the appearance of regional brain atrophy in MCI subjects.

There was no significant correlation observed between the scores on recognition of famous faces and volumetry for any of the cohorts including areas considered important for face recognition, i.e. parahippocampal gyri and fusiform gyri. Functional MRI studies have demonstrated activation of the right fusiform face area in the recognition of famous faces [27]. Another study had also shown the correlation of performance on task for recent famous names with regard to hippocampal volume. This study also did not find a significant correlation with regard to hippocampal volume for remote famous names [28]. Our study used remote famous names, and this might explain the lack of a correlation with hippocampal volumes. Yet the significant correlation of this test with standard verbal-visual memory tests in our study could indicate remote memory deficits for semantically familiar visual images.

The small size of the cohorts cautions against the generalization of our results. The crosssectional design of our study with a lack of follow-up period may have impacted our findings with respect to the evolution of MCI to early AD although the same enables us to intricately characterize intuitive deficits in $\mathrm{MCI}$ at risk of $\mathrm{AD}$. The characteristics of converters from nonconverters could thus not be assessed. MRI in the entire cohort could not be done due to logistic reasons, and this might have affected our correlation analysis as only a subgroup could be systematically studied for neuropsychology-MRI correlations.

\section{Conclusions}

There were significant differences observed between MCI-controls and MCI-AD cohorts with respect to visual paired associative learning on face-name pairs. With reference to FP there were significant differences observed between MCI-control and AD-MCI cohorts. There was good concordance observed between the newly devised test of visual associative learning and results on other standardized measures of delayed recall. The results of the FP test were concordant with measures of delayed recall in the MCI cohort. The newly developed test of visual paired associate learning had a good concordance with reduction in volumes of the right and left cuneus in the AD cohort. There was no significant correlation of the newly developed test of associative learning with atrophy in MCI and the control cohort. Overall these results point towards a rather broad affection of cognition in MCI patients as compared to the initial belief of isolated episodic memory affection. The tests for visual paired associate learning have demonstrated considerable potential in diagnosing latent (early) cognitive impairment in the elderly even prior to the development of MRI volumetric changes, and its potential as a novel neuropsychological biomarker in early cognitive impairment is apparent. 
Nanda et al.: Novel Face-Name Paired Associate Learning and Famous Face Recognition in $\mathrm{MCl}$

\section{Acknowledgement}

This study was conducted in part with a grant-in-aid from the Cognitive Science Initiative, Department of Science and Technology, Government of India (CSRI 90/2012).

\section{Statement of Ethics}

This study had the approval of the Institutional Ethics Committee (reference No. IEC/978/2016), and informed consent was obtained from all cases and controls.

\section{Disclosure Statement}

None of the authors have any other financial/competing interests.

\section{Appendix 1}

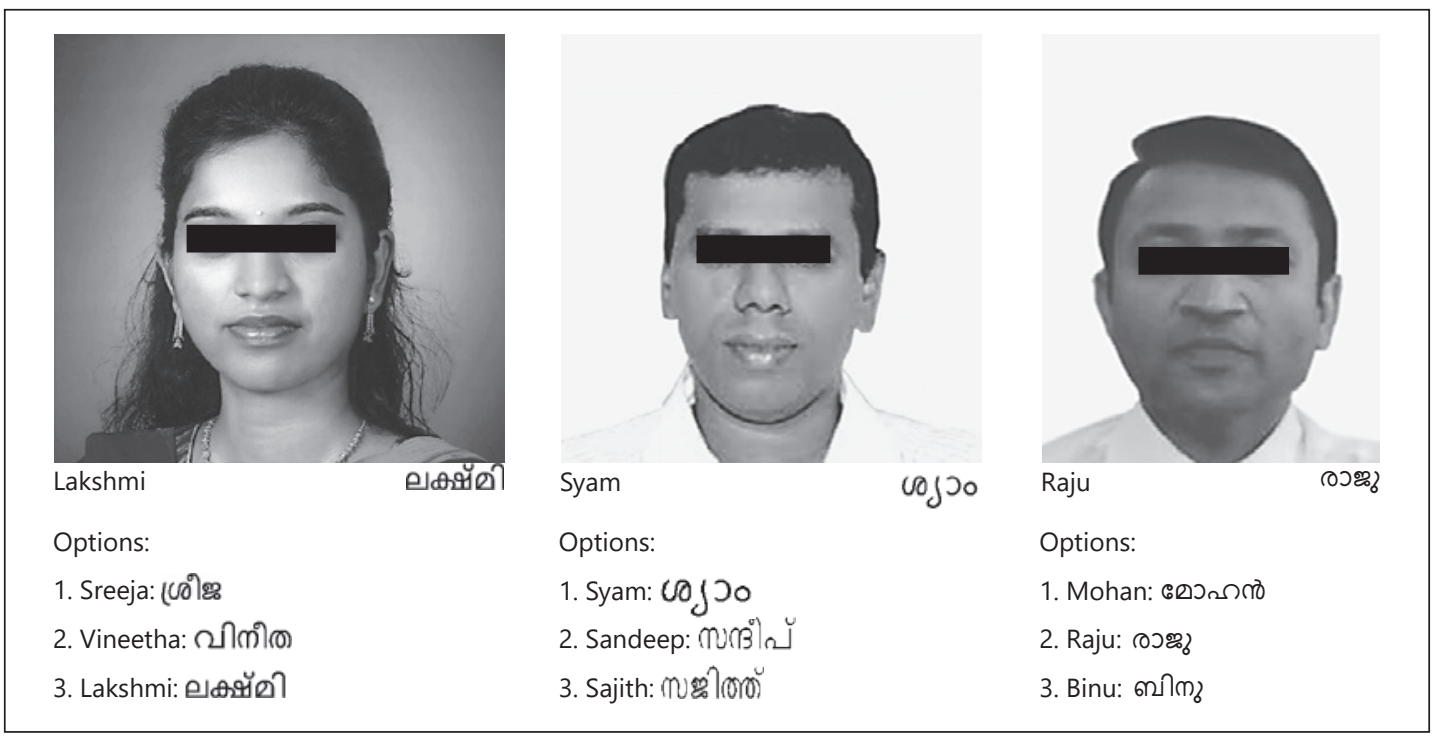

\section{Appendix 2}

\section{MRI Protocol for Acquisition and Post-Processing}

Structural MRI was performed to identify the earliest GM changes seen in AD and MCI. The image acquisition was performed in 3-T GE 750-W Discovery with a standard transmitreceive head coil. In all subjects, MR images of the entire brain were obtained using a 3-dimensional T1-weighted BRAVO sequence (TR = $8 \mathrm{~ms}$; TE = $3 \mathrm{~ms} ; \mathrm{FA}=12^{\circ} ; \mathrm{FOV}=25.6 ; 172$ slices with thickness $1 \mathrm{~mm}$ ).

Image Analysis

All the images were post-processed using CAT12 software in SPM 12 (Wellcome Department of Imaging Neuroscience, London, UK) for the cortical volume measurement. All 
Nanda et al.: Novel Face-Name Paired Associate Learning and Famous Face Recognition in $\mathrm{MCl}$

the images were oriented in the anterior commissure-posterior commissure line. The images were segmented into GM, white matter, and cerebrospinal fluid, and spatially normalized into the Montreal Neurological Institute space using the CAT12 DARTEL procedure with custom settings. The segmentation was followed by modulation for preserving the volume of a particular tissue in a fixed voxel, and volumes of GM, white matter, cerebrospinal fluid, and total intracranial volume were calculated. The resulting images were smoothed with an isotropic Gaussian kernel of $8 \mathrm{~mm}$ full-width half-maximum.

\section{ROI Analysis of Subcortical Volumes}

The segmentation algorithm in the LPBA 40 atlas in the Statistical Parametric Mapping (SPM) atlas was used to estimate the bilateral volumes of hippocampus, parahippocampus, fusiform gyrus, cuneus, and precuneus. The mean values of each region were obtained and tabulated. Group comparison among MCI, AD, and controls were performed by one-way ANOVA within the SPM12 general linear model. Group comparisons were tested with a corrected threshold of $p<0.05$, which determines the clusters with significant differences in GM concentration. Three separate contrasts (NCI vs. MCI, NCI vs. AD, and MCI vs. AD) with age, sex, and total intracranial volume as covariates were used to compare the GM density between participants. The significant atrophic regions were overlaid on T1-weighted standard brain images, allowing the localization of areas of significant GM loss. The atrophic regions are reported in Montreal Neurological Institute coordinates with the help of the xi view toolbox (http://www.alivelearn.net/xjview/).

\section{References}

1 Petersen RC, Roberts RO, Knopman DS, Geda YE, Cha RH, Pankratz VS, et al.; The Mayo Clinic Study of Aging. Prevalence of mild cognitive impairment is higher in men. Neurology. 2010 Sep;75(10):889-97.

2 Bäckman L, Jones S, Berger AK, Laukka EJ, Small BJ. Cognitive impairment in preclinical Alzheimer's disease: a meta-analysis. Neuropsychology. 2005 Jul;19(4):520-31.

3 Bondi MW, Salmon DP, Galasko D, Thomas RG, Thal LJ. Neuropsychological function and apolipoprotein E genotype in the preclinical detection of Alzheimer's disease. Psychol Aging. 1999 Jun;14(2):295-303.

4 Caselli RJ, Reiman EM, Osborne D, Hentz JG, Baxter LC, Hernandez JL, et al. Longitudinal changes in cognition and behavior in asymptomatic carriers of the APOE e4 allele. Neurology. 2004 Jun;62(11):1990-5.

5 Winblad B, Palmer K, Kivipelto M, Jelic V, Fratiglioni L, Wahlund LO, et al. Mild cognitive impairment-beyond controversies, towards a consensus: report of the International Working Group on Mild Cognitive Impairment. J Intern Med. 2004 Sep;256(3):240-6.

6 Belleville S, Fouquet C, Duchesne S, Collins DL, Hudon C. Detecting early preclinical Alzheimer's disease via cognition, neuropsychiatry, and neuroimaging: qualitative review and recommendations for testing. J Alzheimers Dis. 2014;42 Suppl 4:S375-82.

7 Polcher A, Frommann I, Koppara A, Wolfsgruber S, Jessen F, Wagner M. Face-name associative recognition deficits in subjective cognitive decline and mild cognitive impairment. J Alzheimers Dis. 2017;56(3):1185-96.

8 Wagner AD, Poldrack RA, Eldridge LL, Desmond JE, Glover GH, Gabrieli JD. Material-specific lateralization of prefrontal activation during episodic encoding and retrieval. Neuroreport. 1998 Nov;9(16):3711-7.

9 Sperling RA, Bates JF, Cocchiarella AJ, Schacter DL, Rosen BR, Albert MS. Encoding novel face-name associations: a functional MRI study. Hum Brain Mapp. 2001 Nov;14(3):129-39.

10 Ahmed S, Arnold R, Thompson SA, Graham KS, Hodges JR. Naming of objects, faces and buildings in mild cognitive impairment. Cortex. 2008 Jun;44(6):746-52.

11 Petersen RC. Mild cognitive impairment as a diagnostic entity. J Intern Med. 2004 Sep;256(3):183-94.

12 Morris JC. The Clinical Dementia Rating (CDR): current version and scoring rules. Neurology. 1993 Nov; 43(11):2412-4.

13 McKhann GM, Knopman DS, Chertkow H, Hyman BT, Jack CR Jr, Kawas CH, et al. The diagnosis of dementia due to Alzheimer's disease: recommendations from the National Institute on Aging-Alzheimer's Association workgroups on diagnostic guidelines for Alzheimer's disease. Alzheimers Dement. 2011 May;7(3):263-9.

14 Mathuranath PS, Hodges JR, Mathew R, Cherian PJ, George A, Bak TH. Adaptation of the ACE for a Malayalam speaking population in southern India. Int J Geriatr Psychiatry. 2004 Dec;19(12):1188-94. 
15 Menon R, Lekha V, Justus S, Sarma PS, Mathuranath P. A pilot study on utility of Malayalam version of Addenbrooke's Cognitive Examination in detection of amnestic mild cognitive impairment: A critical insight into utility of learning and recall measures. Ann Indian Acad Neurol. 2014 Oct;17(4):420-5.

16 Schmidt M. Rey Auditory Verbal Learning Test: A Handbook. Los Angeles: Western Psychological Services; 1996.

17 Wechsler D. Wechsler Memory Scale (Revised Manual). San Antonio (Texas): The Psychological Corporation; 1987.

18 Monchi O, Petrides M, Petre V, Worsley K, Dagher A. Wisconsin Card Sorting revisited: distinct neural circuits participating in different stages of the task identified by event-related functional magnetic resonance imaging. J Neurosci. 2001 Oct;21(19):7733-41.

19 George A, Mathuranath PS. Primary progressive aphasia: a comparative study of progressive nonfluent aphasia and semantic dementia. Neurol India. 2005 Jun;53(2):162-5.

20 Tombaugh TN. Trail Making Test A and B: normative data stratified by age and education. Arch Clin Neuropsychol. 2004 Mar;19(2):203-14.

21 Warrington EK. Recognition Memory Test. Windsor, U.K: NFER-Nelson; 1984.

22 Warrington EK, James M. The Visual Object and Space Perception Battery. Bury St Edmunds, England: Thames Valley Test Company; 1991.

23 Hampstead BM, Stringer AY, Stilla RF, Deshpande G, Hu X, Moore AB, et al. Activation and effective connectivity changes following explicit-memory training for face-name pairs in patients with mild cognitive impairment: a pilot study. Neurorehabil Neural Repair. 2011 Mar-Apr;25(3):210-22.

24 Amariglio RE, Frishe K, Olson LE, Wadsworth LP, Lorius N, Sperling RA, et al. Validation of the Face Name Associative Memory Exam in cognitively normal older individuals. J Clin Exp Neuropsychol. 2012;34(6): 580-7.

25 Pennanen C, Kivipelto M, Tuomainen S, Hartikainen P, Hänninen T, Laakso MP, et al. Hippocampus and entorhinal cortex in mild cognitive impairment and early AD. Neurobiol Aging. 2004 Mar;25(3):303-10.

26 Pitel AL, Chanraud S, Rohlfing T, Pfefferbaum A, Sullivan EV. Face-name association learning and brain structural substrates in alcoholism. Alcohol Clin Exp Res. 2012 Jul;36(7):1171-9.

27 Axelrod V, Yovel G. Successful decoding of famous faces in the fusiform face area. PLoS One. 2015 Feb; 10(2):e0117126.

28 Seidenberg M, Kay CD, Woodard JL, Nielson KA, Smith JC, Kandah C, et al. Recognition of famous names predicts cognitive decline in healthy elders. Neuropsychology. 2013 May;27(3):333-42. 